\title{
A Unified Theory of Mind-Brain Relationship: Is It Possible?
}

\author{
Shashidhar Belbase \\ College of Education, University of Wyoming, Laramie, Wyoming, USA \\ Email: belbaseshashi@gmail.com, sbelbase@uwyo.edu \\ Received May $11^{\text {th }}, 2013$; revised June $11^{\text {th }}, 2013$; accepted June $18^{\text {th }}, 2013$
}

\begin{abstract}
Copyright (C) 2013 Shashidhar Belbase. This is an open access article distributed under the Creative Commons Attribution License, which permits unrestricted use, distribution, and reproduction in any medium, provided the original work is properly cited
\end{abstract}

\begin{abstract}
The mind-body relationship has vexed philosophers of mind for quite a long time. Different theories of mind have offered different points of view about the interaction between the two, but none of them seem free of ambiguities and questions. This paper attempts to use a mathematical model for mind-body relationship. The model may generate some questions to think about this relationship from the viewpoint of operator theory.
\end{abstract}

Keywords: Philosophy of Mind; Unified Theory of Mind; Operator Theory

\section{Introduction}

This paper argues for possibility of a mathematical model of operator theory of mind-body relationship as a unified theory of mind. At first, I would like to briefly review the existing theories of mind-body interrelation. Different theories of mind (e.g., materialism, dualism, and idealism) have their points of views about correspondence between mind and body. There have been different points of views offered regarding the mind-body relationship. Then, I would like to argue in favor of a mathematical model of a functional operator between mental states and brain states. Finally, I would like to discuss a quantum operator which is possible between mind-brain functional interfaces.

Philosophers have attempted to give a coherent view of the mind-body relationship; however, it seems that none of these endeavors has succeeded in offering a unified explication of this thorny relationship. We need to look no further than a broad outline of the four main theories so far expounded under the categories of monism and dualism to recognize the failure to provide a unified view (Cf. Anderson, 2003). First, Armstrong (1993) has argued for a materialistic theory of the mind, which maintains that the mind can be explained in terms of a universal material law. Thinking, reasoning, believing, remembering, perceiving, and understanding, general capacities of the mind, are nothing but the workings of universal material law. Some philosophers (e.g., Ward, 1894) have organized this law in two distinct forms of organization: chemical organization and biological organization, which are fundamentally based on interactions and organizations among different material substances. The materialistic theory of mind assumes that mental states are reducible to physical states (Robinson, 2012), i.e., monism, and concludes that "primitive reality is material" (Marchal, 2013: p. 2).

Second, some philosophers, perhaps Rene Descartes most famously among them, have argued that the mind and body are two separate substances, i.e., dualism. Cartesian dualism assumes that mind and body cannot be explained in terms of a universal material law because the mind is non-corporeal and non-physical. According to this theory, the mind is non-physical, mental substance and the body is physical, corporeal substance. The body and mind exist separately and have distinct features with distinct properties (Robinson, 2012). Therefore, "dualism assumes two fundamental, non-reducible, realities: mind and bodies, together with some possible associations between them" (Marchal, 2013: p. 2).

There are basically two kinds of dualism: substance dualism and property dualism. Substance dualism considers mental and physical substances as two distinct substances, mental and physical. Property dualism considers mental and physical properties as two distinct kinds of properties in relation to mind and body. Substance dualists claim that mind is non-physical substance that does not have space extension whereas body consists of physical substance that has space extension. Rene Descartes defended this form of dualism and hence it is also known as Cartesian dualism. Property dualists claim that there is only one kind of substance, that is physical or material substance but these substances have two kinds of properties, one is physical properties and the other is mental properties (Howard, 2012).

Third, other philosophers, such as Kant, Berkeley, and Hegel, have emphasized the supremacy of mind over material substance. Call this, broadly speaking: "idealism". According to this view, there is no other realm except the mental realm. On some naive accounts, there is no mind-independent reality. Everything in the world can be viewed as one's mental construct. Proponents of idealism believe in the sovereignty of mind over physical objects (Heil, 2000). Idealism concludes that "primitive reality is mental" (Marchal, 2013: p. 2).

Fourth and finally, there is the view that suggests neither the mind nor the body exists. If the two do not exist, then there is no need to worry about whether the two interact. What we construe to be either mental or physical is mere illusion. It concludes that "the primitive reality is neither material nor mental" (Marchal, 2013: p. 2). Call this: "nihilism".

Hence, the four points of view concern the ontology of mind and the mind-body relationship. Whereas the four predominant theories of mind have attempted to provide an argument show- 
ing how the mind and body interact, very few people have focused upon the relationship itself. The four views have not given us any reason to believe that we ought to be in the business of discussing the interaction if we have not understood what the status of this so-called "relationship" is.

In this context, I would like to briefly discuss the correspondence of mind-body relationship in different philosophical domains. Then discuss eliminative materialism and why this theory failed to explicate bidirectional correspondence of mindbody interaction. As alternative to this view, I propose a mathematical model of mind-brain function space and operators in the space. I argue that such mathematical model at a basic level can help us to understand mind-body interaction. The model is not an effort to solve mind-body problem, but to provide an alternate approach to look at the problem.

\section{Correspondence of Mental and Brain States}

Now I would like to discuss how major philosophical schools view the correspondence between mental and physical states. This can be interpreted in different ways in dualism and monism. Substance dualism claims that mental and physical states are independent of each other and hence there is no direct correspondence between these two states. Property dualists view that there is bidirectional correspondence between mind and body. However, epiphenomenalism rejects the bidirectional correspondence. This view claims that mental states are causal effect of brain states whereas there is no effect of mental states on brain states. Leibniz's view about mind-body relationship is not based on causal relationship, but it is a non-causal correspondence between the two (Kulstad \& Carlin, 2008).

Functionalism, a version of materialism, claims that mental and brain states have causal correspondence. This view compares the mind-body interaction with functioning of a computer. Within this view mind-body relationship can be understood in terms of their functional interaction as input, process, and outcome (Block, 1980). The recent version of materialism, eliminative materialism, does not accept the bidirectional correspondence between mental and brain states (Feyerabend, 1963). This view eliminates the mental state. According to this view, mental state is nothing more than a psychological state and it has no separate existence from the brain state (Churchland, 1981). I would like to discuss eliminative materialism in a separate subheading.

\section{Eliminative Materialism}

Churchland's (1989) eliminative materialism is an extreme kind of monistic material reductionism, which recommends that all folk-psychological talk about the "mind" be eliminated in favor of a kind of neuro-speak. Paul Churchland (1988) has clarified the notion of eliminative materialism in the following way:

$[W]$ hen neuroscience has matured to the point where the poverty of our current conceptions is apparent to everyone, and the superiority of the new framework is established, we shall then be able to set about reconceiving our internal states and activities, within a truly adequate conceptual framework at last. Our explanations of one another's behavior will appeal to such things as our neuropharmacological states, the neural activity in specialized anatomical areas, and whatever other states are deemed relevant by the new theory. Our private introspection will also be transformed, and may be profoundly enhanced by reason of the more accurate and penetrating framework it will have to work with-just as the astronomer's perception of the night sky is much enhanced by the detailed knowledge of modern astronomical theory that he or she possesses (Churchland, 1988: pp. 44-45).

Churchland has claimed that our conception of mental states, such as beliefs, desires, fears, pains, joys, emotions, and attitudes, must be re-conceived. We must eliminate such folk-psychological talk in favor of a more precise alternative derived from a discussion of neuro-physiological changes. There is no such thing as private introspection or separate mental phenomena beyond brain function (Churchland, 1989; Lycan \& Pappas, 1972).

The Churchlands' eliminative materialism may be considered hyper-reductive, in that, atoms, molecules, and neurons play a significant part in the theory of mind (Cf. Bickle, 1997). It is to recognize that "Humans, like everything else in the universe, were at bottom just organized assemblies of atoms, although the organization was neither understood nor assumed to be simple" (p. 240). According to this view, our body is simply an organization of atoms. This kind of materialistic theory of mind assumes that a living system can be continuously reduced into smaller sections (e.g., bodies into systems, systems into organs, organs into tissues, tissues into cells, cells into organelles, organelles into complex molecules such as DNA and RNA). U1timately, on this view, a theory of mind is reduced to a theory of brain and the molecules or neurons that make it up.

Eliminative materialism could not explicate the mind-body interaction in the sense that it failed to provide logic to how mind is reduced to body or how bodies (material substances) organized to form mental states or properties. Eliminating a mental state as a part of folk psychology does not resolve the issue. If we bring consciousness in relation to mental state, eliminative materialism has not clarified how the neurons give rise to consciousness and how the consciousness give rise to mental states with beliefs and desires. It is not yet clear why other theories of mind be eliminated before neuroscience itself provides a convincing explanation for sophisticated mind-body interaction in a subtle way. Its attempt to reduce theory of mind into theory of brain, grossly, fails to establish such interactional correspondence. Therefore, a mathematical model of correspondence between mental and physical states may provide an alternative to this theory for a broader understanding of mindbody interaction. For this, I would like to introduce a very simple mathematical analytical tool to look at correspondence between mental and physical states.

\section{A Mathematical Function}

We can assume mathematical logic as a product of mind and it may represent a mental state. That means when we have the logic of interrelation of two things (either physical or nonphysical) we are in a state of mind in which we experience mental stimulation. The degree of stimulation may depend on the gravity of the logic in terms of its value and consequence in our decision within the logic. The logical interpretation of such and such (e.g., $\mathrm{x}$ is bigger than $\mathrm{y}$ ) is purely a mental. There is no such thing either relational or un-relational (e.g., x bigger than y) beyond our perception and mental construction. Our scheme of "bigger than or smaller than or equal" is a representational and relational one. When we say $\mathrm{x}$ is bigger than $\mathrm{y}$, we observe them or measure them or perceive them and make a 
conclusion. It also depends on relative states of $\mathrm{x}$ and $\mathrm{y}$ during observation or measurement. Our construction of the relationship is subjective and mental even when we claim an objective measurement. Likewise, we can assume different biological, chemical, and physical changes in the brain corresponding to each state of logic. We may have experienced such physiological stress in the brain based on our logic of whether we did something good or bad. The perception of doing something good or bad is our mental state. We may continue sweating. Even we may continue feeling of a headache and a stress on brain. These are real experiences. Therefore, we can see and feel a correspondence between mental state and brain state. Sometimes we can think of this kind of correspondence in terms of mathematical functions.

Now, I can think of a set of all of my mental states constituting a space Ls (i.e., a space of mental states) and a set of all brain states constituting a space Bs (i.e., a space of brain states). Now the question is: Is there a strict correspondence between Ls and Bs? Whether there exists such correspondence may depend on how the two spaces Ls and Bs interact each other. Correspondence theorists (e.g., Benedetti et al., 2010) accept that there is such a relationship between mental and brain states. However, this correspondence can be of two kinds: one is a strict and strong correspondence and the other is a loose and weak correspondence. Therefore, for each element of Ls there is a either strong or weak corresponding element in Bs and viceversa. A strong correspondence is strict one-on-one correspondence where a weak correspondence is a correspondence of one element in a domain (initial space) with more than one element in the range (the final space). This correspondence can be interpreted as both interactional cause and effect relationship or without any causal interaction, which is a parallel correspondence. The correspondence of mental state Ls with brain state Bs shows can be described by function (Figure 1) relations. All such possible functions constitute a space of functions Fs.

The set of all mental states (or logical states) in the space Ls and set of all brain states in the space Bs can be interrelated with the function $\mathrm{F}$ either as onto or into. These onto and into functions can be one-to-one and onto, one-to-one and into, many-to-one and onto, and many-to-one and into.

\section{One-to-One and onto Function}

If the function $\mathrm{F}$ is one-on-one and onto, then for each brain state there is a distinct mental state and vice-versa (Figure 2). We can express this function as $\mathrm{F}(\mathrm{L} 1)=\mathrm{B} 1, \mathrm{~F}(\mathrm{~L} 2)=\mathrm{B} 2, \mathrm{~F}(\mathrm{~L} 3)$ $=\mathrm{B} 3$, and so on. The function $\mathrm{F}$ describes each brain state as a unique feature of corresponding mental state or each mental state as a unique feature of corresponding brain state. This kind of relationship between mental and brain states can be considered as a consistent mind-body relationship. Each mental state (e.g., L1, L2, L3, ...) is paired with a corresponding brain state (e.g., B1, B2, B3, ...). The order may not be the same but the pairing is distinct as in the Figure 2. When $\mathrm{F}$ is one-on-one and onto, then $\mathrm{F}^{-1}$ (inverse of $\mathrm{F}$ ) exists and that maps Bs to Ls. We can predict one's mental state once we know his or her brain state and vice-versa. However, the correspondence of L1 to B1 or B1 to L1 does not mean that they are identical. It is not that $\mathrm{L} 1$ is reduced to $\mathrm{B} 1$ or $\mathrm{B} 1$ is reduced to $\mathrm{L} 1$. Correspondence of $\mathrm{L} 1$ to $\mathrm{B} 1$ or $\mathrm{B} 1$ to $\mathrm{L} 1$ is a non-reductive transformation. In this sense, this correspondence is different from Armstrong's (1993) identity theory.

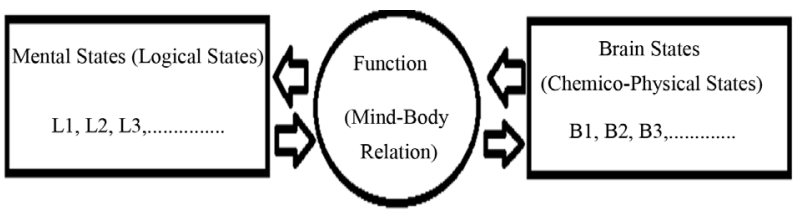

Figure 1.

Functional model of mind-brain correspondence relationship.

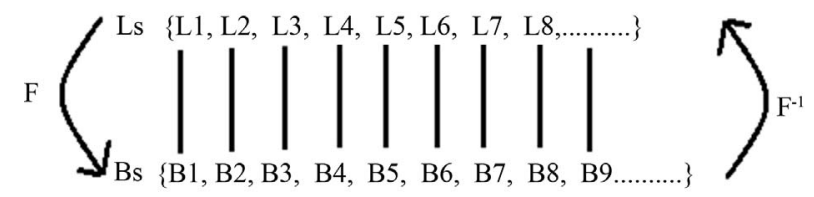

Figure 2.

One-to-one and onto function of mind-brain relationship.

Let's assume that a person has a mental state of pain on his sciatic nerve. For each pain state (i.e., a mental state of being in the pain), he or she has a corresponding nerve problem at the sciatica and then it is responded by his or her brain with a neural firing. Every moment he or she has this kind of pain at sciatic region, there is one-to-one correspondence between mental state (with extreme feeling of the pain) and the severe nerve pressure and corresponding brain state (i.e. a physical state). The mental states and brain states both have this correspondence through a function $\mathrm{F}$.

\section{One-to-One and into Function}

If the correspondence of mental states and brain states is one-on-one into, then for each mental state in Ls there is a unique element in brain state within the space Bs. That means for mental states L1, L2, L3, ... there are brain states B1, B3, $\mathrm{B} 5, \ldots$ but also there are brain states B2, B4, B6, ... not associated with any mental states. Likewise, there may be the relations of brain states B1, B2, B3, ... to mental states L2, L4, $\mathrm{L} 6, \ldots$ but also there are mental states L1, L3, L5, ... not associated with any brain states (Figure 3). From the view point of strict correspondence of mind-brain, that is token identity theory in relation to cause and effect, and then there should not be such state which breaks the one-on-one relationship. However, physicalism has not yet proved strict correspondence between mind and brain (Earley Sr., 2008; Hendry, 2006). Neuroscience has speculation of strict one-on-one correspondence, but it has yet far from the proof. This shows that there are cases which do not obey strict one-on-one relationships (Figure 3). It is like there is a software program (analogous to mind) in a computer but it has no corresponding hardware (analogous to body) or it may have some hardware without software to operate it within the computer. In one-to-one and into case between Ls and Bs there is no strict correspondence between mental and brain states. In such a case, either some brain states or mental states are idle (without pairing) or they do not have active roles in such correspondence.

Let's assume that one has a certain body part like a tail (i.e., a physical state) by mutation, but this does not have any relationship to his or her mental state. When he or she is in a mental state of fear and wants to use that part for safety, he or she has no idea about how to move it and how to use it for safety purposes. Now the physical state of being with a tail-like part is not corresponded to a mental state. He or she even cannot think 
of using it. However, similar organ in some other animals (let's say a dolphin) can be used for navigation. That means a dolphin may make sense of direction within water with this part however a person with such a body part can not.

Likewise, we can suppose that there is a physical entity named "zeta" which we cannot perceive because we lack the appropriate sense organ to know about it. A dolphin, however, can perceive "zeta" because it has, e.g., a "zetan organ". We cannot comprehend "zeta" because we lack the "zetan organ" the dolphin possesses. Just as we cannot experience the zeta, there may be certain physical states that we do not comprehend because we lack the ability to sense it.

\section{Many-to-One and onto Function}

If a function is many-on-one and onto, then for each brain state in the domain there are more than one elements in the mental states, and vice-versa (Figure 4). We can express this function as $\mathrm{F}(\mathrm{L} 1, \mathrm{~L} 2)=\mathrm{B} 1, \mathrm{~F}(\mathrm{~L} 3, \mathrm{~L} 4)=\mathrm{B} 2, \mathrm{~F}(\mathrm{~L} 5, \mathrm{~L} 6, \mathrm{~L} 7)=\mathrm{B} 3, \mathrm{~F}(\mathrm{~L} 8$, L9) $=$ B4, and so on. The function $F$ describes each brain state as a unique feature of corresponding mental states or each mental state as a unique feature of corresponding brain states. This kind of relationship between mental and brain states can be considered as an inconsistent mind-body relationship. Multiple mental states are paired with the same brain states. When $\mathrm{F}$ is many-to-one and onto, then $\mathrm{F}^{-1}$ (inverse of $\mathrm{F}$ ) does not exist (Figure 4). Most possibly, we cannot predict one's mental state once we know his or her brain state and vice-versa.

Let's assume that one's mental state is very unstable. He or she is constantly changing thoughts, beliefs, and values. He or she imagines different kinds of things. These imaginations are not consistent with his or her beliefs and values. Then the person may have multiple mental states that correspond to a certain brain state. This places his or her brain into an immense cognitive pressure. He or she may feel extreme mental pressure and then the brain simply cannot process all those mental events at a time. The inverse brain state to mental state function stops and the brain may stop further working. This may lead to a serious psychological problem leading to unbalanced thinking. The same brain state has now many mental states. The person may be mentally ill.

\section{Many-to-One and into Function}

If a function is many-on-one and into, then for each brain

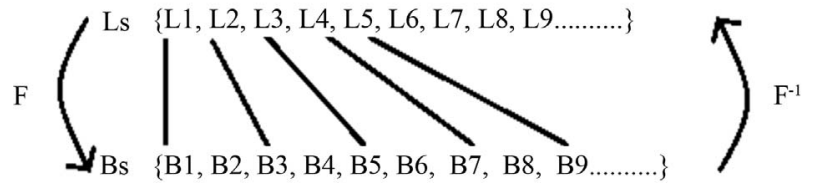

Figure 3.

One-to-one and Into Function of mind-brain relationship.
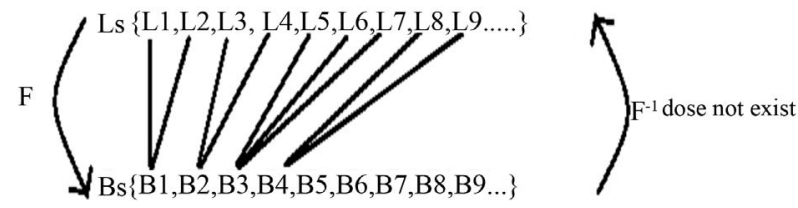

Figure 4.

Many-to-one and onto function of mind-brain relationship. state in the domain there are more than one elements in the mental states, and vice-versa (Figure 5). We can express this function as $\mathrm{F}(\mathrm{L} 1, \mathrm{~L} 2)=\mathrm{B} 1, \mathrm{~F}(\mathrm{~L} 3, \mathrm{~L} 4)=\mathrm{B} 3, \mathrm{~F}(\mathrm{~L} 5, \mathrm{~L} 6, \mathrm{~L} 7)=$ $\mathrm{B} 5, \mathrm{~F}(\mathrm{L8}, \mathrm{L} 9)=\mathrm{B} 7$, and so on. Although all the elements of the domain (Ls) seem to be paired with an element in the range (Bs), but there are some elements (e.g., B2, B4, B6, ...) remained unpaired. The function $\mathrm{F}$ describes each brain state either a feature of corresponding mental states or it just does not involve some elements in Bs in the relationship. This kind of relationship between mental and brain states can be considered as an inconsistent mind-body relationship. Multiple mental states are paired with the same brain states. When $\mathrm{F}$ is manyto-one and into, then $\mathrm{F}^{-1}$ (inverse of $\mathrm{F}$ ) does not exist (Figure 5). Most possibly, we cannot predict one's mental state once we know his or her brain state and vice-versa.

Let's assume that one's mind is in very active state. He or she has several mental states. Despite his or her multiple mental states at a time he or she may be using the brain partially. The multiple thought processes in the mind may not have corresponding brain processes as distinct physical phenomena. Many intelligence powers within the mind are working at the same time, however the brain (the physical part) may not be supportive to all the mental functions. Then many mental states may have correspondence to a few brain states leaving some other brain states even without relating to any mental events.

Above discussion shows that a specific mental state even may have corresponding multiple brain states or vice versa. Then either one mental state may have multiple brain states or one brain state may have multiple corresponding mental states. It is not yet known how the function space Fs actually behaves in the interfaces of Ls and Bs. The function between the mental and brain state interface should be a very sophisticated one due to myriad of interactions going on in both directions - from mind to brain and from brain to the mind. The mental states within Ls and brain states within Bs are not static. Every moment they are changing. The dynamic relationships of Ls and Bs can modify Fs. The space of functions Fs may not be a function in classical mechanics but it also may represent an instantaneous function in continuous flux. Given that it may represent a quantum state of Ls and Bs, the function becomes a quantum function.

Any theory of mind in the past has not succeeded to describe whether such function is specific and certain. In a broader sense, without limiting Fs either in classical or relativistic or quantum state it may represent a holistic concept of mind-body correspondence when extended with a set of operators of infinite dimensions. Because such a condition might exist, we can derive a unified theory of mind in terms of a unified mind-brain function; however, the boundary of the spaces Ls and Bs might be an issue in such a model.
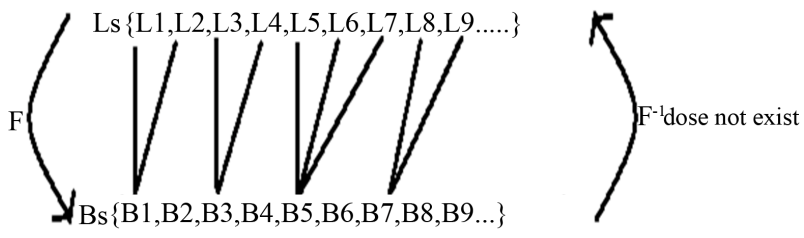

Figure 5.

Many-to-one and into function of mind-brain relationship. 


\section{Complexity with Boundary}

Let's assume that one-dimensional space constitutes lengthwise all functions of the mind and body. The perception of a length or distance between two fixed points through eyes creates a brain state B1 (with neuron firings) and corresponding mental state L1 with the consciousness of the length of an object or distance between two points in the space. The observation of the length creates a schema of distance between two points in the brain. This schema then serves as a basis for mental state of being conscious to the distance. That is why one does not jump down from a height of ten fit thinking that it would harm his or her body. This sense of distance in mind as a mental state and corresponding schema in brain state create such functional relationship of one dimensional space. Hence, the associated functions could be F1 with one dimension (i.e. distance).

We can assume that two-dimensional space constitutes all functions of mind and body associated with the area. The visual perception of an area through eyes creates area schema and a corresponding brain state B2 (with neuron firings). The visual schema of area as an extension of two dimensional space generates an awareness and corresponding mental states L2. This mental state is exhibited with the consciousness of surface area. The function that corresponds B2 to L2 or vice versa could be F2 with two dimensions (i.e. length and width). Again, we can assume that three-dimensional space constitutes all functions of mind and body associated with volume. The schema of volume through visual perception to the brain creates a brain state B3 (with neuron firings). The three dimensional spatial distribution of points in space or three dimensional extension of an object creates an awareness and corresponding mental states L3 with the consciousness of volume. The correspondence of B 3 to L3 or vice versa creates a space of functions F3 with three dimensions (i.e. length, width, and height).

We can continue increasing the dimensions of a space. However, we have no idea if our brain states have such stimuli to make schema of dimensions greater than three. Our awareness of such higher dimensions can continue forming mental schema of such dimensions. That is what we do in mathematics of higher dimensions that physically do not exist. The addition of new dimensions can continue to infinite number (at least theoretically). Some physical dimensions beyond length, width, and height can have brain states in terms of other attributes of objects. However, mentally, we can think of and conceive a space with infinite dimensions.

The mathematically universal set Ls and Bs have their boundaries. Is it possible that the sets Ls and Bs have their corresponding boundaries? In terms of the continuous flux of mental and brain states, it seems that these spaces do not have specific boundaries (i.e. in terms of elements within the spaces). A logic space including all Ls's does not have any upper and lower bounds (i.e. There is no such upper-ness or lower-ness in mental states). Mathematically, we cannot enlist all the elements of Ls in a sequence. Even at a certain point of time, we are unsure of what mental states we are in. It is possible that there are multiple mental states we are in at any given time. Enumerating mental states seems to be an impossible task one ought not to undertake. For example, when we studying for an exam and trying to memorize or to critically analyze important concepts, our mental states cannot be individuated and counted.

Although my brain may have a finite number of neurons at a time, these finite numbers of neurons may have uncountable number of brain states. The brain states are not simply the states of individual neurons, but these states can be considered as ongoing physiological, chemical, and physical processes or changes. It may not be possible to segregate different brain states at the same time. Our brain functions in a very complex way. It can process all perceptual and intuitive information at a time. That means it can process visual, auditory, olfactory, and other retrospective and prospective information at the same time. It may come up with a complex brain state. Hence we cannot expect a clear-cut boundary of sets Ls and Bs, which means these states are fuzzy. The fuzziness of Ls and Bs gives impetus to interpret the mind-body relationship in alternative ways. However, a mathematical model of mental and brain states seems a less fuzzy and, therefore, more palatable interpretation of the mind-body interaction.

\section{Reconceptualizing the Functional Model}

I discussed possibilities of different mental states and brain states in the forms of correspondence relationships. A mathematical functional model of the mind-body relationship is a plausible model because it rests on how Fs is characterized and positioned within different philosophical schools. Idealists, dualists, and materialists all accept some tenable position common in discussions of the mind-body relationship. The debate, however, if my argument is sound, lies in how they understand functions in Fs.

For an idealist, Fs is nothing more than a function in domain Ls with the range Bs where any brain activity is a function of mental activity. The supremacy of Ls over Bs through Fs could be established by characterizing the function space Fs. Like wise, for a materialist, Fs is nothing more than a function in domain Bs within a range Ls and any mental activity is a function of brain activity. A predominant theme in the work of neuroscience is understanding how the brain functions in a way that generates certain mental states (if any).However, they do not seem to recognize the importance of a function when they discuss the body and mind. In my understanding, they have failed to characterize function space Fs or even acknowledged that such a function space Fs exists as a model.

For a dualist (both substance and property dualists), Fs is nothing more than a function space in domain Bs with the range Ls or vice versa. In both cases, Fs may not be equivalent on two independent theories of mind because they are quite different functions due to nature of direction of fit from Ls to Bs or Bs to Ls. For each and every traditional position in the theory of mind, idealism, materialism, and dualism, the main problem lies within the understanding nature of functional space Fs.

Despite the complexity of Fs, the functional space of Fs may yield fruitful results for the mind-body relationship. The one-toone onto, one-to-one into, many-to-one onto, and many-to-one into functions constitute basic mathematical relational properties of domains and ranges in terms of Bs and Ls within the function space Fs, which may provide a basis to generate a mathematical functional model of the mind-body relationship.

Modern neuroscience uses brain-scanning technology (e.g., fMRI) to study brain states. This technology is still not developed well enough for us to say that these scans may form an accurate prediction of such states. For example, we cannot generalize from the results of a brain scan to what Ls and Bs are in ordinary conditions. We may conclude that Ls and Bs merely seem to be just one aspect of the mind-body relationship.

We can purport that both Ls and Bs are real or complex do- 
mains and ranges of functions within a logical space Fs; we can come up with a complex set of all possible functions in Fs (space of mind-brain functions) that complicates the model. $\mathrm{F}_{1}$ is a brain function that maps $\mathrm{L}_{1}$ to $\mathrm{B}_{1}, \mathrm{~F}_{2}$ is another brain function that maps $\mathrm{L}_{2}$ to $\mathrm{B}_{2}$, and we can continue this way to form an innumerable number of brain functions in the space Fs. There is no simple model of function $F$ of mind-brain correspondence that can represent a holistic concept of the mindbody relationship. The non-reductive transformation of brain functions in terms of a mathematical model with a set of functions Fs mapping from Ls to Bs or vice versa is never a complete one. Hence, the unified theory of mind in terms of function $F$ of mind-brain may not help us understand the mind and body relationship in a complete sense. Then we need to conceptualize a new unified theory of mind in conjunction with a broader operation on the functions in Fs.

\section{Conceptualizing an Operator for Functions}

The mathematical functional model of mental states in Ls and brain states in Bs with functions in Fs shows that a unified theory of mind is possible if all the functions are brought together with an operator or operators. The mind-brain correspondence function model is a mathematical model that may include all possible functions Fs of brain states in Bs and mental states in Ls within a set of operators U. Here, an operator, such as $U$, is a mathematical device for a change in the function, in Fs, to which it is applied. We can apply different kinds of operators in relation to mathematical functions. The simple operators are addition, subtraction, multiplication, and division. More complex operators are differentiation, integration, and transformations. In logic, operators are and, or, negation, etc. Each operator employed alters the behavior of the function and gives end results different from the original function. The set of operators $U$ is a model that operates on the functions Fs. Suppose an operator "P" in the set U can transform a function in Fs with a brain state in Bs and a mental state in Ls into a third state, a "deton" state Ds (a form of life-state). The operator P in U (as a unified function operator for all $F_{s}$ ) can be used to translate both brain state and mental state into D. D is a complex life state. The set of operators, U, has, both Bs and Ls as input to produce $\mathrm{D}$ as output. Accordingly the functions with the operators can be considered a holistic theory of mind because $U$ includes all kinds of operators within the space of functions Fs.

Mathematically, the set of operators $U$ is a modulator of all functions, either one-to-one or many-to-one or onto or into functions. We can discuss the model with an analogy to the Hilbert space.

\section{An Analogy to the Hilbert Space}

Analogizing the mathematical functional model set out above to Hilbert space would suffice to describe the operator model of mind-brain functions. A Hilbert space (in mathematics and physics) is an infinite dimensional space of inner products of vectors. Hilbert space is useful to express the status of mindbody system or relationship digitally (Kapteina \& Zhang, 2008), since this space has been used in physics to solve body-body interaction problems. However, there may be a possibility to apply this space to solve mind-body problems. We can assume that the mental events that compose mental space is a vector space including mental states. The mental states as a vector state can be realized in terms of differentials of mental states with a parameter of time. We can imagine an infinite dimensional operator $\mathrm{P}$ in $\mathrm{U}$ within a functional space $\mathrm{Fs}$. The set $\mathrm{U}$ is a set of operators in Hilbert space. The functional space can be an inner product space of $\operatorname{Bs}(t)$ and $\operatorname{Ls}(t)$ where $t$ is a reference point in time. The vector space connotes a physical space but in mathematics it is an ideal space where we can assume both mental and physical vector spaces, then Fs: $\langle\mathrm{Ls}(\mathrm{t})| \mathrm{Bs}(\mathrm{t})>\rightarrow$ Ds $(\mathrm{t})$. The inner product space of mind and brain corresponds to the space of all functions of mental states Ls to functions of brain states Bs.

There are different linear and nonlinear operators in Hilbert space to interpret the nature of different kinds of inner product space. In the mind-body relational space of functions Fs, we can work out analogous operators. These operations have not yet been discussed in the literatures of philosophy of mind and even neuroscience. Now the research in these fields can come up with such operators that can represent interrelation of elements of an inner product space of mind and brain to deton states. Hence, we can imagine a multidimensional (even infinite dimensional) operator P in U for a holistic understanding of mind-body relationship. This kind of model opens the possibility that there can be different operators to describe unique combinations of mental and brain states. This multidimensional operator theory seems to present a unified theory of mind, an alternative to Churchland's eliminative materialism (1989) and Stern's matrix theory of mind and brain (1992).

\section{Quantum Operator in Mind-Body Function}

Our mental states and brain states function within the world of uncertainties. These uncertainties arise due to changes in the time, space, and environment with which both mind and brain interact. This change is continuous. Therefore, any two mental or brain states are not identical. Our mind changes every time and we have never ever experienced two identical thoughts. Likewise, our brain states are changing within every moment due to change in the supply of oxygen and nutrients and also different perceptual and introspective phenomena. Such state of flux of mental state and brain state relates to quantum phenomenon of mind-body relationship. Some scholars, for example Goertzel (1993), tried to relate mental states with quantum physics. He stated that "according to quantum physics, no physical entity is ever in a definite state; the most one can ever say about a given entity is that it has certain probabilities of being in certain states" (p. 133). When we consider differential states of mind and brain with time, Heisenberg's famous uncertainty principle might be applicable to describe such relationship.

The relative change of mental states $(\Delta \mathrm{Ls})$ and brain states $(\Delta \mathrm{Bs})$ can be used as analogous to two components of Heisenberg's uncertainty principle. The degree of change in mental states and brain states can be very small but their product might be greater than a very small possible differential value such as half the Plank's constant divided by pi. Mathematically, $(\Delta \mathrm{Ls})(\Delta \mathrm{Bs}) \geq \mathrm{M}$ and $\mathrm{M}$ might be equivalent to $\mathrm{h} /(2 \pi)$ where $\mathrm{h}$ is Plank's constant. This relationship is analogous to Heisenberg's uncertainty principle for the momentum state and position state of a particle. A question may come in relation to what does this product really represent. Can it be applied to mental states and brain states? To me, mind as such has no separate existence out of our body. Then, it should be something associated with the brain or body. When we take it 
as a part of the body that continues to exist until this body exists, then it should have an existence in the form of a physical entity either as matter or energy or some form of synergy (combination of matter and energy). This synergy may be the actual function Fs that transforms mental states to brain states or brain states to mental states. There is always change in this synergy with $\Delta \mathrm{S}=(\Delta \mathrm{Ls}) \quad(\Delta \mathrm{Bs}) \geq \mathrm{M}$ where $M$ is analogues to $\mathrm{h} /(2 \pi)$ in Heigenberg' principle. When the change in this synergy is zero, then the mind and brain both may possibly collapse. All the functions of mind-brain states with the space Fs can operate in a different way at different situation relative to time and space. The operator $\mathrm{P}$ within the set of operators $\mathrm{U}$ may carry different kinds of transformations in Fs and result into different deton states. We can assume this operator as a quantum operator because it operates on mind-brain functions based on probabilistic modulation of Bs and Ls to Ds. Here, F is mind-brain function of inner product of mental state and brain states. After modulation by an operator P on F, the system within the synergy produces a life state (i.e., deton state) D. Hence the life state of a person is the result of different quantum operations on mind-brain functions in Fs. This model may have some empirical implications for future research in mindbrain relations.

\section{Some Implications}

The operator $\mathrm{P}$ in $\mathrm{U}$ can be thought of having multiple dimensions and hence it can modulate any kind of functions in space Fs into deton state D. We ought to consider some implications of the unified theory of mind-brain functional model. For example, we can consider deton states from one-to-one into and onto functions when operated through any element $\mathrm{P}$ of $\mathrm{U}$. Since the input function converts all brain states into unique mental states and vice versa, the modulated life state with an operator P might be a normal life with a consistent mind-brain relation.

The case of many-to-one onto and into functions when modulated with the operator $\mathrm{P}$ in $\mathrm{U}$ might result in quite different deton states than the one discussed earlier. Here the one mental state may have many corresponding brain states or vice versa. In such a case the modulation of functions in Fs with the operator $\mathrm{P}$ in $\mathrm{U}$ can produce deton states either seriously disturbing mental states or brain states. The modulation of functions that correspond one mental state into multiple brain states can result in a life state that is full of diverse behaviors, e.g., the person may act akratically. Hence, knowledge of different functional interactions between mind and brain and influence of operator $\mathrm{P}$ on these functions might help in understanding the complex mind-body problem.

\section{Challenges}

There are some issues in relation to the model with a set of operators in $U$ as a unified theory of mind-brain relationship. Ontologically, if Ls is purely non-physical and Bs is purely physical, then the functions Fs and its space that relates Ls and Bs might be challenged. Epistemologically, a mathematical function in Fs and its space must be interpreted as a fuzzy space. The non-physical nature of Ls makes the functions Fs as an inner product space of Ls and Bs fuzzy. The operator theory with operators in $U$ might have fuzzy nature due to very subtle correspondence of mental states in Ls and brain states in Bs. Alternately, if both Ls and Bs are physical, then the model with functions Fs as inner product of Ls and Bs vectors and the operators in U seem more viable. Since both Ls and Bs are physical states and can be modulated with operators in $U$ on functions in Fs, then it can be seen as parallel to infinite dimensional Hilbert spaces. There will be a direct analogy of space of functions Fs with the Hilbert space. Maybe, the function space Fs may have better interpretability and applicability analogous to other spaces beyond Hilbert space (e.g., Probability space).

Philosophers can work with mathematicians, neuroscientists, and physicist to uncover more about such spaces within which all mental states and brain states can be better understood and predicted in terms of mathematical models of observables and state variables. More properties and interrelations analogous to Hilbert space and others have to be studied further in order to develop such a unified theory of mind and brain. This model has many implicit and explicit challenges of identifying the appropriate mode of functional correspondence between mental states in Ls and brain states in Bs through an operator in U.

\section{New Direction}

The fluctuation of mental states in Ls and brain states in Bs may or may not be predicted by a model depending on the nature of the model and associated variables within it. The predictability of mental or brain states depends on the strength of the functional model and associated operator. The functional model of mind-brain relationship has to go through this examination. If a model functions well with a wide range of possibilities, then it could be a viable model, though it may not represent the reality as it is. A model is not a reality. It is only a way to describe the relationship between state of mind and brain in this case. Operator model on functions within a space of mental and brain states analogous to Hilbert space may open a possibility of new interpretation of mind-body relationship. Nonetheless, this model needs further attention from philosophical and scientific community either in the similar form or a different takeover.

\section{Acknowledgements}

I would like to acknowledge Joseph Ulatowski for providing constructive feedback and comments on an earlier draft of this paper, which was written for his class, Topics in the Philosophy of Mind. I would like to thank anonymous reviewers for encouraging comments/feedback to the manuscript.

\section{REFERENCES}

Anderson, J. (2003). Visions of mind and body: A technological solution to the mind-body problem. England: The University of Reading. Armstrong, D. M. (1993). A materialist theory of the mind. New York: Routledge.

Benedetti, G., Marchetti, G., Fingelkurts, A. A., \& Fingelkurts, A. A. (2010). Mind operational semantics and brain operational architectonics: A putative correspondence. The Open Neuroimaging Journal, 4, 53-69.

Bickle, J. (1997). Psychoneural reductionism: The new wave. Cambridge, MA: The MIT Press.

Block, N. (1980). Introduction: What is functionalism? In N. Block (Ed.), Readings in philosophy of psychology (Vol. 1, pp. 171-184). Cambridge, MA: Harvard University Press.

Churchland, P. (1981). Eliminative materialism and the propositional attitudes. Journal of Philosophy, 78, 67-90. http://dx.doi.org/10.2307/2025900

Churchland, P. (1988). Matter and consciousness (Revised ed.). Cam- 


\section{S. BELBASE}

bridge, MA: The MIT Press.

Churchland, P. S. (1989). Neurophilosophy: Toward a unified science of the mind/brain. Cambridge, MA: The MIT Press.

Earley Sr., J. E. (2008). How philosophy of mind needs philosophy of chemistry. International Journal for Philosophy of Chemistry, 14, 126.

Feyerabend, P. (1963). Mental events and the brain. Journal of Philosophy, 40, 295-296. http://dx.doi.org/10.2307/2023030

Goertzel, B. (1993). The structure of intelligence: A new mathematical model of mind. New York, NY: Springer-Verlag. http://dx.doi.org/10.1007/978-1-4612-4336-6

Heil, J. (2000). Philosophy of mind: A contemporary introduction. New York: Routledge.

Hendry, R. F. (2006). Is there downward causation in chemistry? In D. Baird, E. Scerri, \& L. McIntyre (Eds.), Philosophy of chemistry: Synthesis of a new discipline (pp. 173-189). The Netherlands: Springer. http://dx.doi.org/10.1007/1-4020-3261-7_9

Howard, R. (2012). Dualism. The Stanford encyclopedia of philosophy. (Winter 2012 Edition).

http://plato.stanford.edu/archives/win2012/entries/dualism/
Kapteina, H., \& Zhang, C. L. (2008). Music therapy in the viewpoint of biophysics. International Journal of Modelling, Identification and Control, 5, 205-209. http://dx.doi.org/10.1504/IJMIC.2008.023122

Kulstad, M., \& Carlin, L. (2008). Leibniz's philosophy of mind. The Stanford Encyclopedia of Philosophy (Fall 2008 Edition). http://plato.stanford.edu/archives/fall2008/entries/leibniz-mind/

Lycan, W., \& Pappas, G. (1972). What is eliminative materialism? Australasian Journal of Philosophy, 50, 149-159.

Marchal, B. (2013). The computationalist reformulation of the mindbody problem. Progress in Biophysics and Molecular Biology. http://dx.doi.org/10.1016/j.pbiomolbio.2013.03.014

Robinson, H. (2012). Dualism. In E. N. Zalta (Ed.), The Stanford encyclopedia of philosophy (Winter 2012 Edition). http://plato.stanford.edu/entries/

Stern, A. (1992). Matrix, logic, and mind: A probe into a unified theory of mind and matter. New York: Elsevier Science Publishing Company Inc.

Ward, L. F. (1894). A monistic theory of mind. The Monist, 4, 194-207. http://www.jstor.org/stable/27897135 http://dx.doi.org/10.5840/monist18944212 